\title{
TV/Series
}

1 | 2012

Les Séries télévisées américaines contemporaines :

entre la fiction, les faits, et le réel

\section{Jules César au secours des scénaristes américains : \\ l'incipit de la série Rome}

Julie Gallego

\section{(2) OpenEdition}

\section{Journals}

Édition électronique

URL : http://journals.openedition.org/tvseries/1057

DOI : $10.4000 /$ tvseries. 1057

ISSN : 2266-0909

Éditeur

GRIC - Groupe de recherche Identités et Cultures

Référence électronique

Julie Gallego, « Jules César au secours des scénaristes américains : l'incipit de la série Rome », TV/

Series [En ligne], 1 | 2012, mis en ligne le 15 mai 2012, consulté le 18 juin 2020. URL : http://

journals.openedition.org/tvseries/1057 ; DOI : https://doi.org/10.4000/tvseries.1057

\section{(c) (i) (9)}

TV/Series est mis à disposition selon les termes de la licence Creative Commons Attribution - Pas d'Utilisation Commerciale - Pas de Modification 4.0 International. 


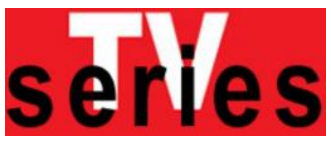

Jules César au secours des scénaristes américains : l'incipit de la série Rome

Bruno Heller, co-producteur exécutif et principal scénariste de Rome, a utilisé comme base pour la série un épisode de La guerre des Gaules (V, 44, 1-14), racontant l'histoire de Vorenus et Pullo. César ne consacrant à ces deux légionnaires qu'un peu plus de deux cents mots, il a fallu, pour une série d'une vingtaine d'heures, puiser aussi dans l'imagination. Néanmoins, ce texte latin est bien le ferment qui va donner corps à la série. La rivalité qui anime ces deux légionnaires les pousse constamment, selon César, à l'action militaire d'exception. Dans la série, cet orgueil militaire valorisé par César est présent dès la première apparition des héros, lors de la bataille d'Alésia. Mais, alors que, chez César, ils apparaissaient comme des doubles, qui par essence même ne pouvaient être départagés par leur vertu militaire, incarnant même l'idéal de uirtus à la romaine, Vorenus devient, dans la série, le supérieur de Pullo, et leurs caractères se singularisent et se heurtent. Cette rivalité, devenue amitié, est le fil conducteur qui tisse la série, dès l'épisodepilote : chez César, leur ressemblance crée un conflit qui les rapproche ; dans la série, c'est leur différence de caractères qui joue ce rôle. Il faut y voir également la transposition de l'évolution des relations entre César et Pompée, mais à l'échelle des simples soldats et du populus et avec une trajectoire inverse: de l'amitié à la guerre. César, dont on connaît la tendance à la déformation historique, a inséré cet épisode dans un but précis, asseoir son prestige de vainqueur de la guerre des Gaules face à Pompée, au début de la guerre civile. Il ne faut donc pas chercher dans son récit la réalité historique : cet exemplum est déjà une réécriture de l'Histoire à des fins politiques et stratégiques. Les scénaristes de Rome étaient donc à bonne école : si les héros ne sont pas fictionnels puisque César les cite, il faut néanmoins leur donner une histoire pour permettre au téléspectateur de pénétrer plus facilement dans l'Histoire ; ce seront des passeurs faits de chair, de sang et de sentiments, mais provisoires, car le personnage central de la série est une abstraction, plus difficile à représenter, ne naissant implicitement que de la superposition des rôles principaux, secondaires et de la masse du populus : c'est la ville de Rome elle-même, le monde romain, comme le montre l'étude du générique et du premier épisode.

i Bruno Heller, co-producteur exécutif, est le principal scénariste de $\mathrm{S}$ Rome, il a su trouver un allié de choix dans l'écriture de sa série Caius Julius Caesar himself. Il est donc temps maintenant de rendre à César ce qui est à César et de faire une petite version latine pour qui veut panem et circenses, ou plutôt du sexe et du sang !

En effet, c'est bien un épisode de La guerre des Gaules qui a servi de base à l'histoire, puisque nous y retrouvons un certain Titus Pullo et un certain Lucius Vorenus, qui prennent les traits du Britannique Ray Stevenson et de l'Écossais Kevin McKidd sur le petit écran. César ne consacre à ces deux légionnaires qu'un peu plus de deux cents mots : pour une série d'une vingtaine d'heures, on comprend aisément que la matière n'était pas suffisante et qu'il a fallu puiser ailleurs, notamment dans l'imagination! Néanmoins, ce texte latin est bien le ferment qui va donner corps à la série, comme nous allons essayer de le montrer, en confrontant le texte de César à quelques passages de la série, et tout particulièrement au 
générique et à l'épisode-pilote de la première saison, constituant l'incipit de la série.

\section{Rome, série HBO : de la diffusion TV au support DVD}

Arrêtons-nous, tout d'abord, sur quelques données techniques importantes pour remettre dans son contexte télévisuel, artistique et commercial la série Rome ${ }^{1}$. La réalisation a eu lieu en 2004 et 2005 et la diffusion en 2005 et 2006 sur la chaîne câblée HBO (en collaboration avec la BBC) ; cette chaîne présente plus d'audace dans ses choix artistiques que les chaînes normales, avec un slogan qui repose sur un démarquage de la télévision traditionnelle : It's not TV, it's $H B O$. Son créneau est plus proche de celui du cinéma, avec parfois même plus d'originalité :

Rome évoque tour à tour les dramatiques télévisées des années 70 où l'on disait la vérité crue sans se défaire du romanesque (I, Claudius) et les marges hallucinées du cinéma bis de la même époque (Caligula). La série prend naturellement sa place dans le line-up 'Histoire' de la chaîne câblée, [...] (avec) une entreprise de démystification qui regarde là où personne ne daigne poser les yeux ${ }^{2}$.

Ce sont des séries qui comptent généralement de 10 à 12 épisodes " soignés ", d'une durée de 50 à 55 min. Mais ce ne sont pas des épisodes «formatés »: pas de cliffhangers, pas de «résumé de l'épisode précédent » ; l'unité majeure est la saison, pas l'épisode, ce qui permet de constituer une sorte de long film d'une dizaine d'heures sous la forme d'un feuilleton, mais qui n'exclut pas une forte cohérence et une très bonne construction au niveau de la sous-unité qu'est l'épisode.

L'executive producer de Rome est Bruno Heller ; il est donc à la fois le directeur artistique, le créateur et le scénariste principal de la série qu'il dirige ; il a scénarisé la plupart des épisodes de la saison 1 et certains de la saison 2 ; il est le créateur de la série The Mentalist, actuellement diffusée sur CBS. L'autre scénariste important de la série, avec William J. MacDonald, est John Milius, qui fut le scénariste d'Apocalypse now et de Conan le Barbare. Michael Apted est le réalisateur de l'épisodepilote (season premiere), il exécute donc les choix du scénariste; il est aussi producteur consultant. C'est un réalisateur de films de cinéma ( $L e$ Monde ne suffit pas, Le Monde de Narnia 3, etc.). Son travail sur Rome lui permet d'être sacré meilleur réalisateur de l'année par la Directors Guild of America.

${ }^{1}$ Thibaut de Saint-Maurice, Philosophie en séries [saison 1], coll. « Culture pop », Paris, Ellipses, 2009 [p. 53-62 sur Rome]

${ }^{2}$ Benjamin Rozovas, «L'alternative HBO », Technikart, article du o1 octobre 2005 <http://www.technikart.com/archives/5646-lalternative-hbo> , consulté le 13 juin 2011. 
Ce que vise la chaîne avec cette série de qualité, outre le nombre de téléspectateurs, c'est la vente des coffrets DVD, une fois la série diffusée. Et les ventes ont, semble-t-il, été très bonnes. Mais pas suffisamment pour qu'une troisième saison puisse voir le jour, tant les deux premières saisons de ce péplum ${ }^{3}$ télévisuel (tournées en Italie, dans les studios de Cinecittà) ont coûté cher.

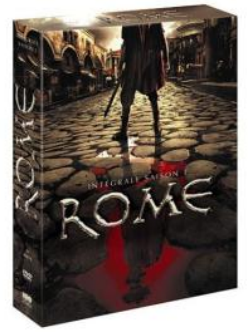

Fig. 1

Fig. 2

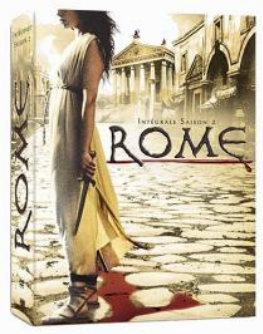

Observons un instant ces DVD énigmatiques (Fig. 1 et 2). Nous voyons un homme armé, anonyme, et son reflet dans une mare de sang : c'est un soldat, debout sur les pavés d'une rue déserte, dont on voit quelques bâtiments ordinaires en arrière-plan ; sur cette image s'inscrit un titre sobre, avec seulement les indications commerciales indispensables. Les mêmes principes sont retenus pour la conception du coffret de la saison 2 mais avec le choix du blanc, face au noir angoissant de la saison 1, et toujours le sang, mais avec une arme, tenue cette fois par une femme inconnue ; les bâtiments choisis sont plus imposants et plus officiels pour la saison 2. Ces deux images ont été utilisées par HBO pour la promotion de la série mais avec une réintégration de la femme dans le décor sombre, plus sanglant (Fig. 3 et 4 ).

\footnotetext{
3 Sur la série TV Rome comme renouvellement du péplum de cinéma : Claude Aziza, Guide de l'Antiquité imaginaire. Roman, cinéma, bande dessinée, Paris, Les Belles Lettres, 2008 ; du même auteur, Le Péplum, un mauvais genre, coll. " 50 questions », Paris, Klincksieck, 2009. Hervé Dumont, L'Antiquité au cinéma : vérités, légendes et manipulations, Paris, Nouveau Monde éditions - Lausanne, Cinémathèque suisse, 2009.
} 


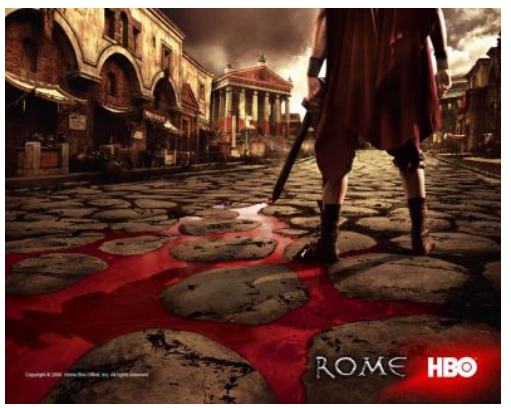

Fig. 3

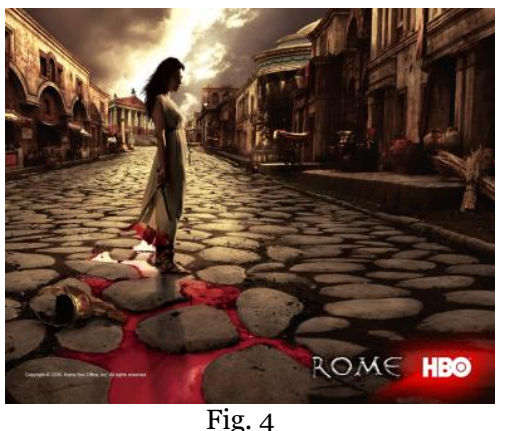

Fig. 4

Il existe deux coffrets pour l'intégrale des deux saisons : l'un reprend ces deux images, l'autre (Fig. 5) accentue encore le dépouillement de la présentation, puisque le titre est seulement encadré par une bordure en mosaïque et par l'insertion de l'aigle de la légion romaine, l'image à bords perdus donnant l'impression d'un mouvement pour amener l'emblème vers Rome. Les coffrets sont en cohérence avec le générique: ni les personnages, ni les acteurs, ni les concepteurs de la série, ni même un moment précis de la série ne sont mis en avant. Juste le personnage principal, Rome, comme dans la dernière image du générique (Fig. 6).

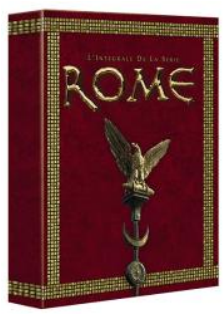

Fig. 5

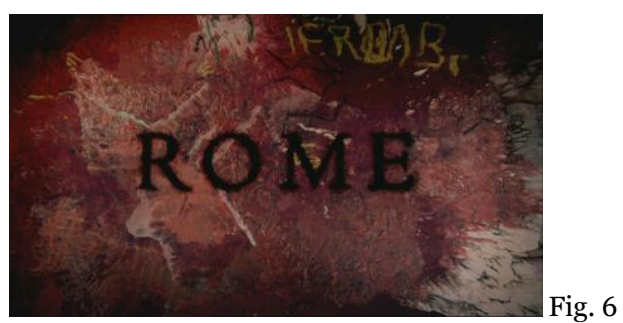

\section{Rome, entité vivante : analyse du générique}

Avant chaque épisode, il s'agit de montrer, par l'utilisation de symboles, et de faire ressentir, par la bande-son évoquant un ailleurs et un autrefois très différents 4 , que Rome, abstraction personnifiée, est le personnage central de la série dans une société pré-chrétienne violente et passionnée, une Rome diverse, hétérogène, loin de son artificialité romantique. Heller a voulu faire de Rome une entité vivante, comme il le revendique dans le bonus L'ascension de Rome. Dès le début, nous entrons

${ }^{4}$ Interview de 2006 du compositeur Jeff Beal par Dan Goldwasser sur Sountrack.net, <http://www.soundtrack.net/features/article/?id=201>, consulté le 13 juin 2011. 
dans Rome par un zoom avant sur l'orbite du crâne d'une mosaïque animée (Fig. 7)5. Nous passons de l'autre côté du miroir, dans le royaume des morts, dans le passé. Cette mosaïque, qui pose une symbolique forte pour toute la série en raison de sa place à l'ouverture ${ }^{6}$, est directement inspirée de la mosaïque polychrome Memento mori de Pompéi (I ${ }^{\text {er }}$ siècle, Musée national d'archéologie de Naples7), œuvre décorative pour le triclinium d'une maison pompéienne, dont tous les symboles, détaillés sur la Fig. 8 concourent à montrer la fragilité de la vie et l'instabilité de la condition humaine : la réalisation des destins des différents personnages manifeste aisément l'égalité devant l'ironie de la mort et de la Fortuna, que l'on soit puissant ou misérable ${ }^{8}$ dans la série.

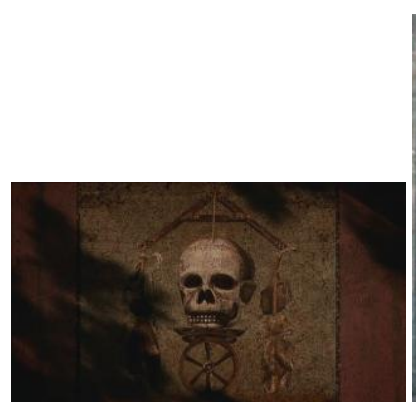

Fig. 7

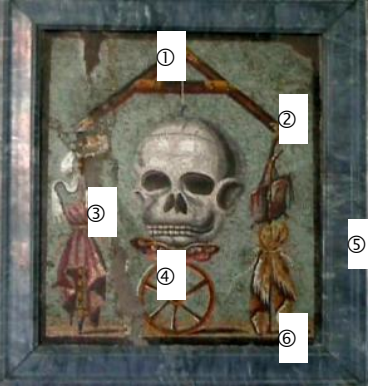

Fig. 8

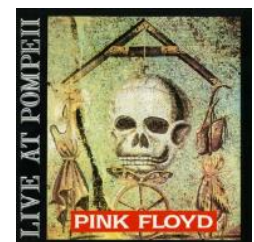

Fig. 9

(1) niveau en A et fil à plomb du charpentier - (2) crâne grimaçant de la mort - (3) sceptre et manteau de pourpre des puissants - (4) papillon de l'âme - (5) bâton et besace du miséreux - (6) roue de la fortune

Par le motif commun d'un arrondi, le mouvement de caméra vers le bas symbolise le fait que la série va s'intéresser au peuple, incarné d'abord par des passants anonymes, et non des personnages de l'h(H)istoire. Le nom des acteurs s'inscrit peu à peu et, en premier, Kevin Mc Kidd-

\footnotetext{
${ }^{5}$ Les séries I, Claudius (BBC, 1976) et Roman Mysteries (2007-2008, BBC One) utilisent aussi des mosaïques en ouverture : une mosaïque de Claude avec un serpent qui ondule, pour la série ancienne, et une mosaïque combinée à une carte de l'empire romain, pour la série jeunesse.

${ }^{6}$ La mosaïque Memento mori réapparaît au moment où César se prépare à affronter Pompée (1 .7), dans le décor en arrière-plan, comme une œuvre d'art personnelle que possède César et qui souligne combien la fortune décide du sort de toute bataille.

7 Waldemar Déonna, « Ex-voto déliens (Pl. xxvii) », Bulletin de correspondance hellénique, 1932 vol. 56 [p. 410-49o]: "Le sens général de ce décor est le même que celui des vases romains ornés de squelettes, usités dans les festins ; ceux-ci rappelaient la communauté de la mort, la brièveté de la vie et l'opportunité d'en jouir avant qu'il ne fût trop tard. Ici, le crâne annonce la mort ; la roue, l'instabilité du sort et des biens ; le niveau en $\mathrm{A}$, puisqu'il sert à mesurer, à niveler, l'égalité de tous devant la destinée. » (p. 463).

Nous remercions Patricia Carles pour nous avoir permis de reproduire ici gracieusement sa photographie, <http://locipompeiani.free.fr/pages/mosaiques.html>, consulté le 13 juin 2011. ${ }^{8} \mathrm{La}$ référence à cette mosaïque passe peut-être par le film du concert Live at Pompei de Pink Floyd (Fig. 9), comme le signale Michel Eloy, <http://www.peplums.info/pep39b.htm> (note 5), consulté le 13 juin 2011.
} 
Vorenus, associé à une scène banale dans la rue. On passe ensuite, avec Ray Stevenson-Pullo, à un combat de personnages en armes. Puis l'actrice qui joue Attia, la femme forte de la série, est associée à la déesse de la guerre. La caméra va tourner dans tous les sens, d'un mur à l'autre, d'une rue à l'autre, sans que nous sachions exactement où nous allons, comme s'il fallait saisir le moindre recoin de Rome pour s'approcher d'une certaine vérité, mais la réalité de ce qu'est Rome ne nous sera pas totalement dévoilée, comme nous l'annoncent toutes les images floues. Puisqu'il s'agit, dans cet article, d'étudier le passage de l'écriture d'un épisode littéraire que César consacre à deux soldats à la constitution d'une série télévisuelle bâtie sur l'entité «Rome», nous ne détaillerons pas toutes les images du générique, mais seulement celles qui annoncent la confrontation d'individualités entre elles ou de groupes entre eux, ou celles qui s'articulent autour du sexe et de la violence. Ainsi peut-on reconnaître sans doute le rapt des Sabines par Romulus et ses hommes, événement fondateur pour la descendance romaine. Puis on discerne le dessin d'un volatile, vraisemblablement une allusion à l'anecdote historique célèbre des oies sacrées du Capitole (Fig. 10), associée ici au peuple et au sexe ${ }^{9}$, comme, quelques séquences plus loin, un homme pourvu d'un sexe disproportionné (Fig. 11), au-dessous duquel on peut lire le graffiti $A R M A^{10}$. Nous voyons ensuite un mur chatoyant sur lequel se détache la dangereuse Gorgone Méduse en 2D (Fig. 12) ; elle réapparaît quelques plans plus loin sur une mosaïque en $3 \mathrm{D}$ (Fig. 13), semblant encore plus vivante et prête à tuer. L'écran est ensuite occupé par un crucifié ensanglanté. Le bâton avec des serpents tenu par un vieillard est interprétable normalement de façon positive, comme le caducée du guérisseur, mais l'inscription MORTEM vient nuancer cette interprétation puisque la mort rode (Fig. 14). C'est au tour de Minerve de faire exploser le haut du crâne de Jupiter pour pouvoir en sortir, scène surmontée du graffiti VITAM, « la vie » (Fig. 15), avec la même association paradoxale que précédemment pour le caducée et la mort. Une scène plus calme prend alors provisoirement place avec des fleurs que l'on entraperçoit, jusqu'au moment où surgit le dessin d'un

9 Populus, «peuple »; futuimus, «nous avons baisé ». Il n'est pas anodin de voir associé à cet épisode le personnage de Marc-Antoine (joué par James Purefoy), dont l'amour pour Cléopâtre le conduira à trahir le peuple romain et à y laisser la vie.

${ }^{10}$ Arma signifie « instruments ; armes, hommes armés » et par extension « la guerre ».

L’organe masculin était un symbole de la fertilité et, à ce titre, "porté en procession lors de nombreuses cérémonies religieuses de l'Antiquité, afin de stimuler la fécondité de la terre, des troupeaux et des hommes, et de prévenir l'extinction de la descendance » (Margaret C. Howatson dir., Dictionnaire de l'Antiquité. Mythologie, littérature, civilisation, coll. "Bouquins », Paris, Robert Laffont, 1998, p. 747-748). La fonction apotropaïque du phallus est bien attestée dans l'art et la société romaine ; elle se trouve ici réinterprétée en un sens guerrier et sexuel.

Lorsque Pullo, prisonnier pour insubordination envers Vorenus, dessine un sexe au début de l'épisode 1, cette valeur sacrée se double d'une valeur plus triviale car c'est à la fois pour s'occuper et pour se concilier les faveurs des dieux qu'il agit ; comme il le clame lui-même plus loin, il ne pense qu'au sexe ... et à la nourriture : "Moi, j'ai des goûts simples ! J'aime tuer mon ennemi, prendre son or et ensuite ses femmes. Et c'est tout» (1.1) Nous sommes loin des questionnements éthiques, religieux et politiques de Vorenus. 
serpent, qui semble onduler sur le mur, symbole du génie tutélaire de tout homme, apprécié dans la société romaine mais nettement déprécié par la tradition judéo-chrétienne qui en fait un animal malfaisant : il est donc perçu comme un élément inquiétant par le téléspectateur. La louve capitoline, le symbole même de Rome, entre alors en scène, et nourrit les jumeaux Romulus et Remus, qui s'entretuent ensuite pour fonder la Ville. Avant le titre sur fond rouge sang, signant la fin du générique et le début de l'épisode 1 (Fig. 6), on découvre la mosaïque d'un calendrier, reprenant des moments-clés de l'agriculture et de la chasse, présentés de droite à gauche pour figurer la remontée dans le temps, et instaurant une présentation cyclique, éternelle, du temps et des conflits humains. Beaucoup de ces représentations ne se laissent pas facilement déchiffrer, même au ralenti. La caméra fait généralement face à ce qui est filmé ${ }^{11}$, à hauteur d'homme ou très près de la terre, avec toujours comme idée de montrer une Rome colorée par la boue, le sang et les détritus, et non une Rome hollywoodienne de marbre blanc.

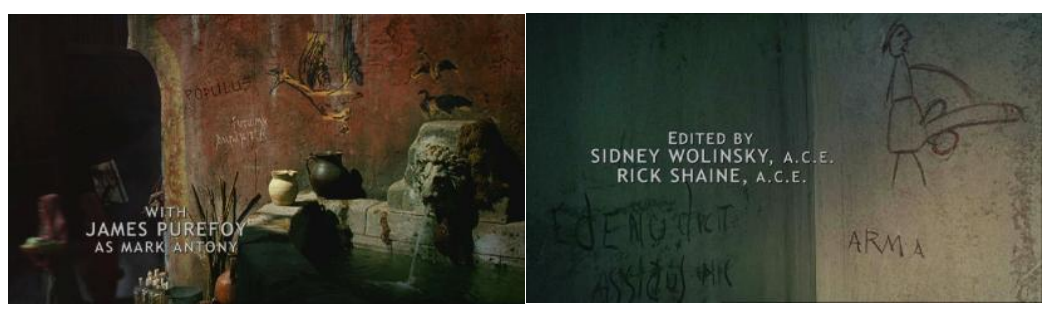

Fig. $10 \quad$ Fig. 11
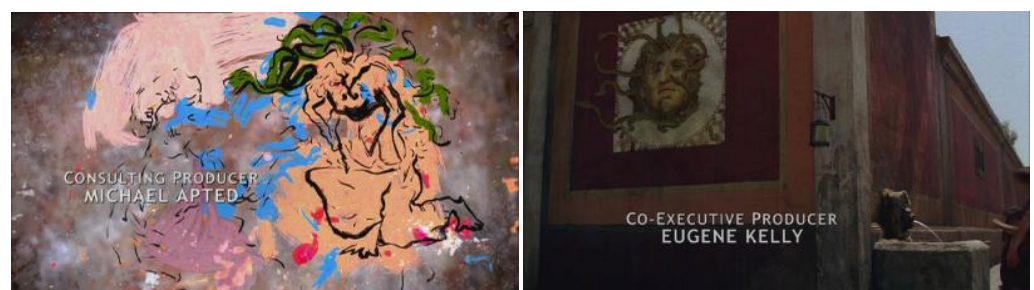

Fig. 12

Fig. 13

${ }^{11}$ Michel Eloy note que le réalisateur « lors des défilés historiques - le triomphe de César, par exemple - préfère placer ses caméras au niveau de la populace, avec des individus qui passent devant, plutôt que de filmer d'en haut, avec de vastes plans d'ensemble dégagés. ", $<$ http://www.peplums.info/pep39g.htm>, consulté le 13 juin 2011. En effet, nous accédons au triomphe d'abord par les yeux de la plus jeune fille de Vorenus, trop petite pour tout voir. 

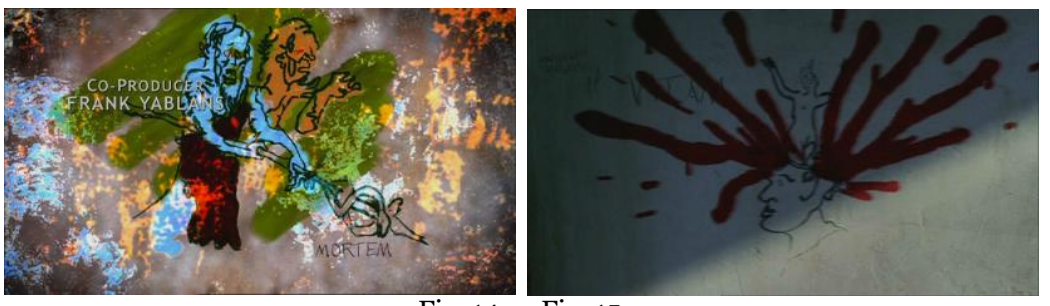

Fig. 14

Fig. 15

Dans le docu-fiction français Le Destin de Rome 1 - Venger César ${ }^{12}$, l'influence de Rome est visible dans le générique, qui prend place au milieu du long résumé de la situation et qui repose sur un rapide parcours du dédale de Rome et de ses sombres ruelles (Fig. 16 à 18). Comme dans la série américaine, les murs servent de support à l'insertion des informations majeures, à savoir les noms du réalisateur et des scénaristes. La caméra est alors également placée à moins d'un mètre de hauteur et filme à plusieurs reprises le pavage.

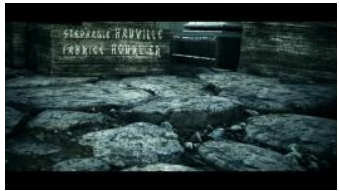

Fig. 16

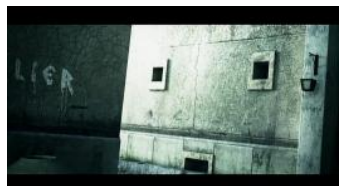

Fig. 17

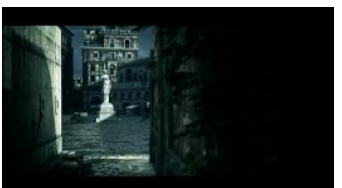

Fig. 18

Avant de nous intéresser au premier épisode de Rome, il faut revenir sur une scène du générique que nous avons délibérément laissée de côté jusqu'à présent. Elle apparaît après l'allusion aux oies du Capitole et à Marc-Antoine (Fig. 10). Il s'agit de l'image sur laquelle va apparaître l'acteur shakespearien Ciarán Hinds, alias César (Fig. 19) : un mur abîmé recouvert de graffitis (mots et dessins).

${ }^{12}$ Le Destin de Rome 1 - Venger César (réal. Fabrice Hourlier ; scén. Stéphanie Hauville et Fabrice Hourlier ; prod. Sylvie Barbe - Docside Production - Indigenes Productions, Arte, 2011, $52 \mathrm{~min})$. 


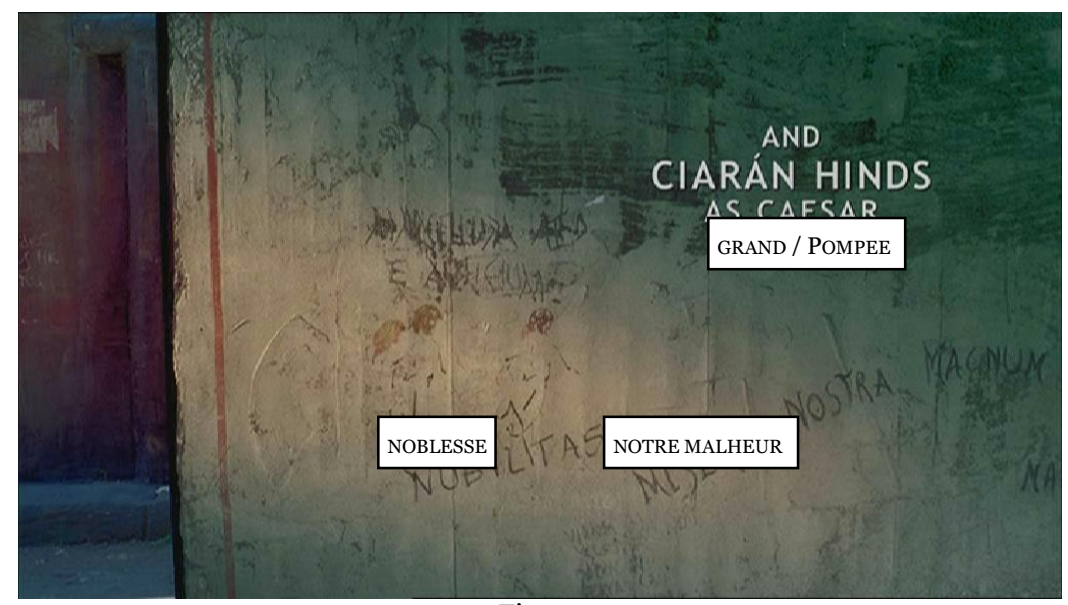

Fig. 19

Ces quelques mots latins font sens dans le contexte de la série : NOBILITAS (qui renvoie au conflit entre patriciens et plébéiens), suivie de MISERIA NOSTRA, autonome ou faisant suite à NOBILITAS ( « La noblesse est notre malheur » ou peut-être « il y a la noblesse, alors que nous nous sommes bien malheureux »); et MAGNUM qui peut être simplement l'adjectif "grand" ou bien le surnom courant de Gnaeus Pompeius Magnus (Pompée le Grand). Tous ces éléments semblent déjà nous lancer dans la guerre civile entre Pompée et César, qui occupe le début du premier épisode.

\section{That's what you missed on... Rome : résumé de l'amitié et des conflits entre César et Pompée}

Passons maintenant à l'analyse de l'abrégé d'Histoire romaine présent au tout début de l'épisode et qui raconte, en quelques phrases, le second triumvirat et les prémices de la guerre civile, en parfaite continuité avec la fin du générique : après la mention du titre apparaît une carte de l'Italie (Fig. 20), qui nous montre déjà que la série Rome ne concerne pas que la ville mais l'Empire; puis la voix off fait un bref résumé de la situation politique. Le statu quo du triumvirat Pompée-Crassus-César est déjà dépassé puisque le consul Crassus est mort en Orient : la voix off ne l'évoque donc même pas. Nous sommes à l'émergence de la dualité entre César et Pompée, qui évoluera en duel, comme le montrent clairement le choix des images, leur apparition soulignée par la voix off et la manière même dont elles sont amenées à l'écran. 


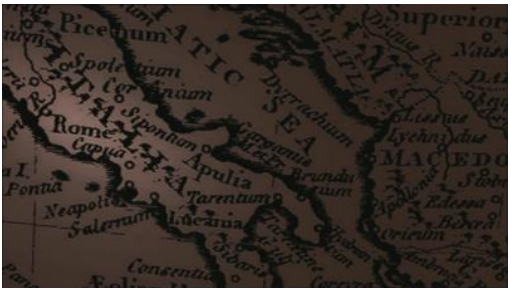

Fig. 20

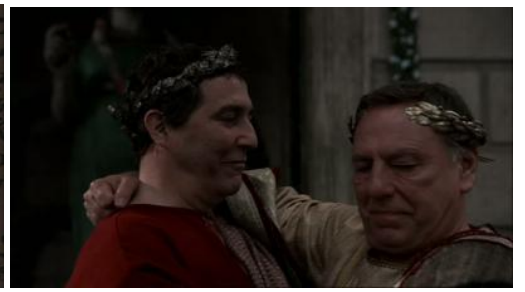

Fig. 21

Le temps de l'amitié et de l'alliance politique entre les deux consuls (Fig. 21) est révolu, l'élément déclencheur (plus proche du prétexte que de la véritable raison) étant la mort de la fille de César, mariée à Pompée, comme Octave l'explique, dès leur première rencontre, à Pullo, à Vorenus ... et au téléspectateur ${ }^{13}$.

Tel un fragment du story-board de la bataille à venir, le dessin, par une main anonyme, de deux guerriers tout aussi anonymes prend alors tout son sens : le combat pour le pouvoir n'a ni temps, ni lieu, ni figure. Et Rome peut être incarnée par des entités différentes mais les combattants Pompée et César (saison 1), puis Marc-Antoine et Octave (saison 2), et surtout Pullo et Vorenus (saisons 1 et 2) en seront le fil conducteur, les piliers principaux faisant avancer la narration. On peut observer ci-dessous (Fig. 22) deux exemples de graffitis pompéiens ${ }^{14}$, qui ont pu inspirer les concepteurs de ce début d'épisode pour la Fig. 23.

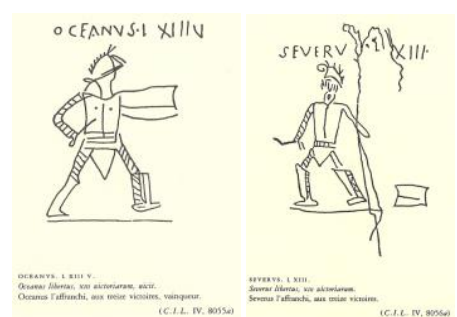

Fig. 22

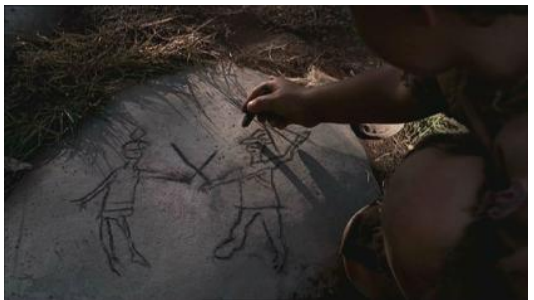

Fig. 23

Les premiers graffitis apparus après la mosaïque Memento mori représentaient également des combattants et c'est sur ces images que s'affichaient les noms des acteurs incarnant les rôles de Vorenus, Pullo et Attia.

\footnotetext{
13 Octave analyse la situation : César se fiche bien de l'Aigle qui lui a été volée et il attend que Pompée attaque le premier. Pullo ne comprend pas l'enjeu politique de ces explications stratégiques d’Octave puisqu'il s'exclame avec naïveté : « Non ! Ils sont comme deux frères ! ». Octave lui répond que la mort de Julia a brisé le lien qui les unissait. (1.1)

14 Philippe MOREAU, Sur les murs de Pompéi. Choix d'inscriptions latines, coll. « Le cabinet des lettrés ", Paris, Le Promeneur, 1996 (réed. Gallimard, 1993).
} 
Voici la transcription en français de cette première séquence d'exposition, tel le prologue des pièces antiques ${ }^{15}$ :

\begin{tabular}{|c|c|}
\hline IMAGES & TEXTE DE LA VOIX OFF \\
\hline $\begin{array}{l}\text { Carte de l'Italie } \\
\text { (1) }\end{array}$ & $\begin{array}{l}400 \text { ans après que le dernier roi fut chassé de la cité, } \\
\text { la république de Rome domine de nombreux } \\
\text { peuples, mais ne parvient pas à se dominer. }\end{array}$ \\
\hline $\begin{array}{l}\text { Dessin des } \\
\text { combattants } \\
(2)\end{array}$ & $\begin{array}{l}\text { La cité est ravagée par des conflits incessants entre } \\
\text { la plèbe et la noblesse. }\end{array}$ \\
\hline $\begin{array}{l}\text { César / Pompée } \\
\text { (3) }\end{array}$ & $\begin{array}{l}\text { Le pouvoir est partagé et l'ordre est maintenu par } \\
\text { deux soldats, deux vieux amis, Pompée le Grand } \\
\text { et Jules César }\end{array}$ \\
\hline $\begin{array}{l}\text { Pompée } \\
\text { (4) }\end{array}$ & $\begin{array}{l}\text { Il y a longtemps, la primauté de Pompée était } \\
\text { reconnue par tous. }\end{array}$ \\
\hline $\begin{array}{l}\text { Pompée + César } \\
(5)\end{array}$ & $\begin{array}{l}\text { Mais après huit ans, pendant que Pompée maintient la } \\
\text { paix à Rome, }\end{array}$ \\
\hline $\begin{array}{c}\text { César } \\
(6)\end{array}$ & $\begin{array}{l}\text { César fait la conquête de la Gaule, le rendant encore } \\
\text { plus riche et populaire. }\end{array}$ \\
\hline $\begin{array}{l}\text { César + combats }+ \\
\text { carte It. }(7)\end{array}$ & $\begin{array}{l}\text { Le pouvoir est en train de changer de main, } \\
\text { et la noblesse a peur. }\end{array}$ \\
\hline $\begin{array}{l}\text { César } \\
(8)\end{array}$ & $\begin{array}{l}\text { Bien qu'il soit de sang noble, César prend le parti de la } \\
\text { plèbe. } \\
\text { Un homme comme lui, un aristocrate à la tête d'une } \\
\text { armée, riche et aimé du peuple,... }\end{array}$ \\
\hline $\begin{array}{l}\text { Écran noir } \\
\quad(9)\end{array}$ & ... pourrait se faire couronner roi \\
\hline
\end{tabular}

Tabl. 1

La première phrase de ce tableau fait à nouveau de Rome le personnage central, par personnification : la Ville agit et elle éprouve des sentiments. On passe en (2) à la vision de deux camps : la plèbe et la noblesse, et les représentants de chaque camp, Pompée et César, avec César qui, en bon animal politique, fait reposer sa conquête du pouvoir sur deux atouts (un changement de camp politique et les campagnes en Gaule ${ }^{16}$ ). Il est présenté en (8) et (9) comme une menace possible pour la république romaine, un nouveau Tarquin le Superbe, d'où son assassinat à la fin de la saison 1. On notera que si César et Pompée se répartissent en (3) équitablement la place à l'écran, César va occuper l'essentiel des autres images : en (5), son image

${ }^{15}$ Voyez, par exemple, le prologue du lare de la maison dans l'Aulularia (La comédie à la marmite) de Plaute.

${ }^{16}$ Il s’agit précisément pour lui de rejoindre le parti des populares contre les optimates, malgré ses origines, et de se servir de ses campagnes en Gaule pour accroître non seulement sa crédibilité de général mais aussi ses finances, qui lui permettront d'asseoir ses ambitions politiques. 
en gros plan va même se superposer à celle de Pompée en plan rapproché (Fig. 24) pour le faire disparaître totalement. En (7), ce sont des images des combats en Gaule et de la carte de l'Italie qui s'ajoutent à celle de César, donnant au spectateur omniscient à voir dans les pensées du consul (Fig. 25) : l'Italie comme objectif et l'armée comme moyen de la conquérir.

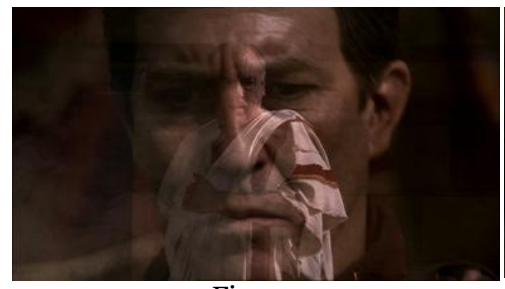

Fig. 24

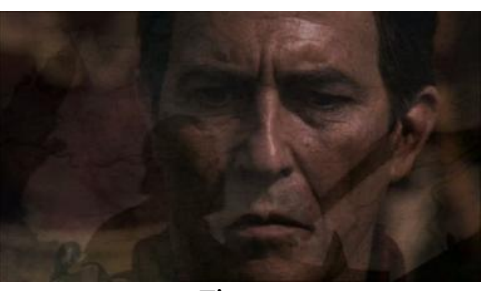

Fig. 25

Après un écran noir, puis l'image de César seul, couronné de lauriers, et un nouvel écran noir sur lequel se termine le discours de la voix off, servant à souligner l'ambition de César et sa marche triomphante vers le pouvoir, la transition se fait naturellement vers le duo Pullo-Vorenus et l'épisode commence véritablement, in medias res, avec la tête casquée de Vorenus ${ }^{17}$ qui surgit du champ de bataille. Il y a donc une assimilation partielle entre le duo Pullo-Vorenus et César-Pompée, entre ces hommes du peuple et leurs dirigeants, afin de montrer que l'Histoire n'est pas faite seulement par les grands dirigeants mais aussi par les «petits », fût-ce malgré eux (la responsabilité de Vorenus est ainsi involontairement engagée dans la mort de Pompée puis de César, celle de Pullo dans la mort de Cicéron et dans la naissance de Césarion). C'est le reflet d'une modification de l'image que nous avons désormais des différentes strates sociales ${ }^{18}$. Si le péplum traditionnel s'intéresse plutôt à ceux qui sont en

${ }_{17}$ Du point de vue de la clarté de la narration et de la cohérence de la fiction, le héros doit pouvoir être clairement distingué par le spectateur. Vorenus portera donc un casque particulier, avec un cimier transversal peut-être anachronique (cf. Silvano Mattesini, Les Légions romaines. L'armement à travers mille ans d'histoire, Rome, Gremese, 2006, p. 77 et 85; ou, au contraire, Philippe Richardot, Les Erreurs stratégiques des Gaulois face à César, coll. " Campagnes \& stratégies », Paris, Economica, 2006, p. 121). Sur ces questions de la représentation de la réalité historique dans la fiction, on pourra se reporter à la polémique née sur Internet :

Florence Dupont, "L'Antiquité falsifiée: 'Rome', ton univers impitoyable », Le Monde diplomatique, article paru d'abord dans la presse en avril 2007 puis repris sur le site Internet, <http://www.monde-diplomatique.fr/2007/04/DUPONT/14645\#nb2>, consulté le 13 juin 2011. Denys Corel et Antoine de Froberville, "'Rome'... malheur à celui qui n'a pas compris. De la confusion des genres entre fiction et Histoire ", 4 juin 2007, <http://denyscorel.overblog.com/article-10713193.html>, consulté le 13 juin 2011. Dupont (5 juin 2007) et Corel (7 juin 2007), <http://denyscorel.over-blog.com/article-23558260.html>, consulté le 13 juin 2011. Voir aussi Florence Broizat, « La véritable histoire de 'Rome' », Télérama, 27 juin 2007 [p. 26-28].

${ }^{18}$ Cf. Sylvaine Bataille, "Haunted by Shakespeare : HBO's Rome », in Television Shakespeare : Essays in honour of Michèle Willems, éd. Sarah Hatchuel et Nathalie Vienne-Guerrin, coll. « ERIAC », Mont-Saint-Aignan, Publications des Universités de Rouen et du Havre [p. 219- 
haut de l'échelle qu'en bas, Rome opte pour une démarche bien différente : " démythifier les grands personnages et réhabiliter le peuple ordinaire19 ».

Un parallèle intéressant peut être fait avec le prologue de Cléopâtre (réal. Joseph L. Mankiewicz, 1963, 4ho8), qui raconte la fin de la lutte entre César et Pompée :

Il arriva qu'à Pharsale, la puissance et la grandeur de Rome s'affrontèrent. César décima les légions de Pompée. Désormais, il était seul maître de Rome. Mais cette fois, son triomphe était sans joie... car les morts que ses légions brûlaient ou enterraient étaient leurs compatriotes.

CESAR. La fumée d'un Romain est aussi noire et son odeur aussi âcre. C'est Pompée qui l'a voulu. Que cela soit retenu.

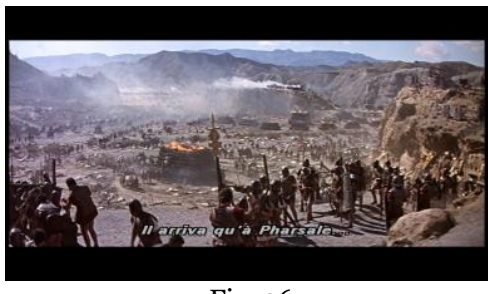

Fig. 26

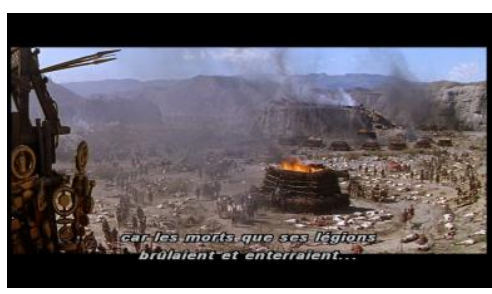

Fig. 28

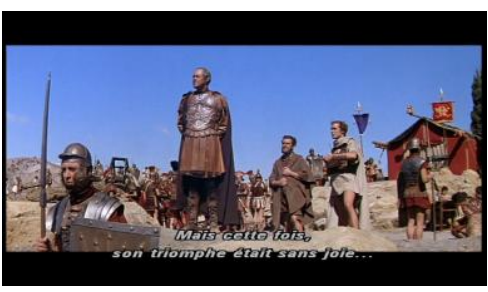

Fig. 27

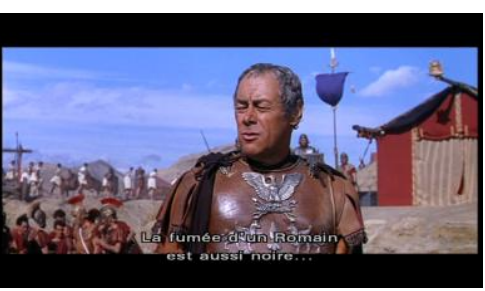

Fig. 29

Les deux parties du film sont centrées respectivement sur César puis sur Marc-Antoine, comme les deux saisons de la série. La série commence quatre ans avant le film (Alésia 52 a.C. et Pharsale 48 a.C. ${ }^{20}$ ), lorsque les

249] : " The series regularly stresses that the privileges held by the aristocrats are not justified by their nature : the nobles are shown as not in essence better than the rest of the population (p. 223). L'auteur analyse les liens étroits que la série entretient avec deux pièces shakespeariennes, Julius Caesar et Antoine et Cléopâtre, pour se démarquer d'une "Holy Rome ». Nous partageons son point de vue sur la fiction historique : il ne faut pas réduire sa richesse à une analyse comparative entre réalité et fiction (p. 246) et même un livre d'historien est une re-construction de la réalité.

${ }_{19}$ D’après Bataille, p. 226 : "demythifying the great personages and rehabilitating the common people. »

${ }^{20}$ La version de 1999 de Franc Roddam (2h57) commence en 47 a.C. - donc après la mort de Pompée et juste avec l'entrée de César à Alexandrie - et le résumé de la situation historique et 
luttes intestines larvées se sont transformées en guerre ouverte entre les deux camps. Une différence de taille, révélatrice de la modification de perspective adoptée dans la série, réside dans le fait que le premier personnage qui prend la parole dans le film après ce résumé est César : la voix off est synchronisée avec un plan éloigné du champ de bataille (Fig. 26) et de la crémation des morts, avant l'apparition d'un plan rapproché sur César, lorsque son point de vue sur la bataille est donné par le narrateur (Fig. 27) puis d'un gros plan lorsque sa voix se fait entendre ${ }^{21}$ (Fig. 28 et 29).

À titre de comparaison, une longue synthèse de près de quatre minutes ouvre Le Destin de Rome, selon une technique narrative propre au genre didactique qu'est le docu-fiction. Nous pénétrons dans la narration par le biais d'un zoom avant sur une carte de l'empire romain (Fig. 30 et 31), lors de l'assassinat de Jules César :

Moins 44 avant Jésus Christ: l'empire romain, immense, s'étend autour de la méditerranée. Jules César s'est nommé dictateur à vie. Il concentre tous les pouvoirs et se comporte comme un roi. Ce nouveau régime déplaît à une grande partie des sénateurs, attachés à la République..

La caméra donne ensuite une vue en surplomb de la Ville (Fig. 32 et 33). Ce résumé par la voix off sera suivi d'un discours de plusieurs minutes d'Antoine au Sénat, analysant la situation politique à la mort du dictateur. Les deux épisodes sont ponctués de résumés en off de ce type, faits par un narrateur omniscient, dont la présence valide comme non fictionnelles les données filmiques.

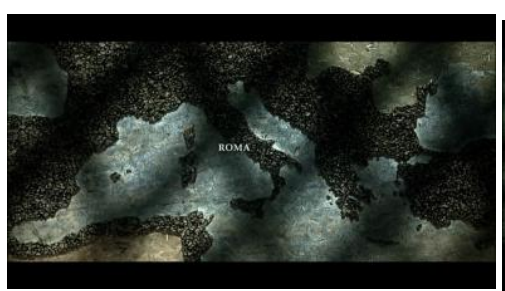

Fig. 30

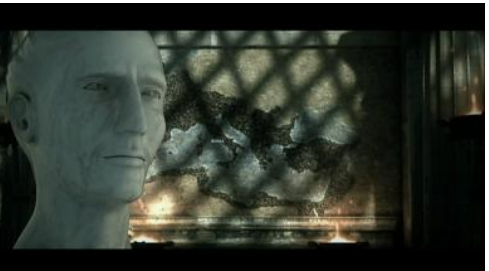

Fig. 31

politique est simplement affiché à l'écran. Il n'y a pas de parallélisme, dans la construction du film, entre les périodes de guerre civile sous César et Pompée et sous Antoine et Octave.

${ }^{21}$ Quelques minutes plus tard, un résumé du même type est fait pour la situation de Cléopâtre en Égypte. 


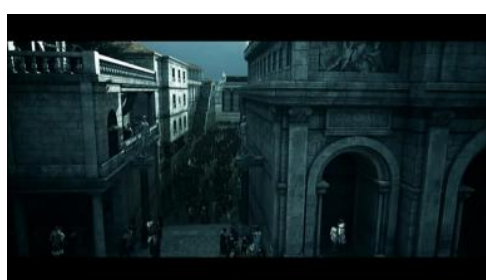

Fig. 32

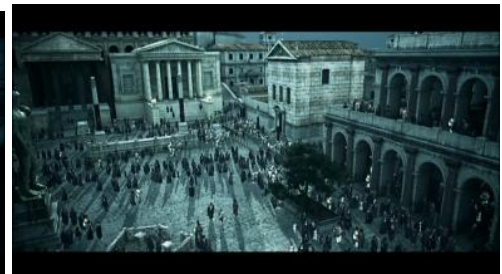

Fig. 33

Dans la série Rome, on passe des plans rapprochés sur Pompée et sur César à un gros plan sur Vorenus et la caméra s'éloigne peu de la troupe pour donner à voir le reste de la bataille : pas de grand plan d'ensemble de la plaine gauloise, le téléspectateur est au cœur de la légion, mêlé dès le début au combat de Pullo, Vorenus et des autres légionnaires. La narration de la voix off est réduite au minimum nécessaire pour comprendre les grandes lignes de la situation.

\section{Qui pour incarner Rome ? Conflits et amitié entre Pullo et Vorenus, personnages de fiction télévisuelle}

Si la série a pour but ambitieux de raconter Rome dans son ensemble, qui retenir pour incarner cette entité démesurée ? Combien de personnages, quels faits, quelles périodes choisir? Selon Vincent Colonna ${ }^{22}$,

le nombre de personnages que peut renfermer un héros collectif n'est pas indifférent. Il y a des limites à l'attention et à la mémoire humaine, surtout avec un média comme la télévision, qui n'est pas associé à l'effort. En fait, il semble bien qu'au-delà de six personnages importants, qui ont leur propre regard sur l'action et leur propre intrigue, le public ait du mal à suivre. [...] Ces comptages montrent quelques invariants, quelques formules dominantes :

- une prédilection pour les groupes de quatre personnes ;

- une moyenne maximum de six/sept personnages principaux ;

- un système tournant qui permet de montrer une moyenne de six protagonistes dominants, mais en renouvelant leur identité.

Dans la série Rome, on a ainsi Vorenus, Pullo / César, Pompée : deux groupes de deux. Auxquels s'ajoutent Attia, Octave, Marc-Antoine : sept personnages majeurs, dont trois personnages secondaires très importants ;

${ }^{22}$ Vincent Colonna, L'art des séries télé ou comment surpasser les Américains, Paris, Payot, 2008, p. 164. 
mais on a aussi Brutus, Servilia et Octavie. Puis, après la mort de Pompée et de César, il y a un basculement entre la saison 1 et 2 pour arriver à Octave et Marc-Antoine, qui montent d'un degré dans la hiérarchie des personnages. Le rôle secondaire de Cléopâtre est également développé dans la saison 2.

Pullo et Vorenus ont a priori existé mais la série leur prête une vie qu'ils n'ont pas eue. Ils permettent de pénétrer à la fois dans l'intimité d'acteurs du pouvoir soumis à une réalité historique et dans l'intimité de gens ordinaires, purement ou partiellement fictionnels, selon un principe largement utilisé dans la plupart des bandes dessinées historiques, et sans que le point de vue soit seulement interne.

Dès leur entrée dans la fiction, le téléspectateur comprend que Vorenus est celui qui maintient l'ordre dans l'armée et Pullo le militaire ingérable. Un premier conflit violent les oppose (en plein combat contre l'ennemi !) et Vorenus voit sa légitimité décroître par l'attitude de Pullo, qui a désobéi à ses ordres. Selon Michel Eloy, "Vorenus et Pullo sont les deux facettes du caractère romain. Le respect des lois - le droit des armes ${ }^{23}$ », ce qui est proche de la présentation de Pompée, d'un côté, et de César, de l'autre. Vorenus et Pullo, deux soldats d'abord en opposition frontale, deviendront amis, en un parcours inverse de celui de Pompée et César.

Deux chevauchées sont constitutives du $\mathrm{duo}^{24}$ : le départ contraint et laborieux en début d'épisode puis le retour volontaire et triomphal, comme si la série, après un premier début avec le résumé sur Pompée et César, puis un deuxième début avec la naissance de l'amitié entre Pullo et Vorenus et le retour sur Rome, pouvait commencer pour de bon après cet épisode-pilote, qui amène Vorenus et Pullo dans l'Histoire : ils accèdent alors au statut individuel de héros dans l'histoire et s'accomplissent en tant que duo, malgré des relations fluctuantes : "Toi et Pullo étiez comme Castor et Pollux ", se souvient un ancien de la XIII ${ }^{\mathrm{e}}$ légion, venu voir Vorenus (1.11). Dans la deuxième saison, le parallélisme apparait même dans les moments tragiques puisque Pullo et Vorenus perdent leurs familles en même temps: la femme de Pullo et son bébé meurent tragiquement et Vorena trahit son père et lui annonce qu'elle, sa sœur et son frère le haïssent ${ }^{25}$, le tenant pour seul responsable de la mort de leur mère Niobé.

La série oscille entre la présentation de leurs différences et de leurs ressemblances, génératrices tantôt de conflits, tantôt de complémentarité.

\footnotetext{
${ }^{23}$ Michel Eloy, <http://www.peplums.info/pep39g.htm>, consulté le 13 juin 2011.

24 Denys Corel, sur son blog Struggling writer, fait un lien avec le personnage du chevalier et celui de l'écuyer du Septième sceau de Bergman, <http://denyscorel.over-blog.com/article23558260.html>, consulté le 13 juin 2011.

${ }^{25}$ Ce qui provoque le départ de Vorenus avec Marc-Antoine, alors que Pullo reste dans le camp d'Octave : ce sont aussi les tensions politiques du troisième triumvirat qui séparent les deux amis.
} 
Vorenus est ainsi « un véritable Catonien » selon Octave. Comparant les caractères de Pullo et Vorenus et leurs différentes opinions politiques exprimées plus tôt dans la discussion (Pullo est prêt à suivre César s'il « envahit » Rome, tandis que Vorenus souhaite s'en abstenir parce que ce serait enfreindre la loi), Attia s'étonne : « On ne vous imagine pas amis ».

VORENUS. Nous ne sommes pas amis. Je suis son supérieur.

PULLO. Uniquement par le rang, et rien d'autre.

VORENUS. Ça suffit, légionnaire! (1.2)

Ce qui semble unir Pullo et Vorenus, comme le constate César qui a l'œil sur cette divinité, c'est la protection que leur accorde manifestement la déesse Fortuna. César devrait punir Pullo pour avoir gardé pour lui l'or de Pompée, qu'il a retrouvé par hasard, et pour ne pas lui en avoir fait part immédiatement. Mais le général constate que la déesse capricieuse accompagne souvent Pullo et se ravise : " Je ne cherche jamais querelle à la chance. Et, en ce qui te concerne, elle t'a visiblement adopté !» (1.4). De même en 1.7 , César ne peut que constater que la chance continue à guider Pullo et Vorenus. Il se sent donc forcé de faire preuve de mansuétude envers Vorenus, qui a relâché Pompée, alors qu'il était à sa merci. Ce qu'Antoine ne comprend pas : " On devrait faire de lui un exemple, au contraire ! » L'imperator explique alors sa décision :

Je n'hésiterais pas avec un autre. Mais ce sont eux qui ont retrouvé l'aigle qu'on m'avait volée ; ce sont eux qui ont survécu à un naufrage qui a décimé toute une armée et qui ont trouvé Pompée en personne sur une plage. De puissants dieux sont, à l'évidence, à leurs côtés. Et moi, je refuse de tuer quiconque peut se prévaloir de telles amitiés.

En 2.6, c'est Pullo lui-même qui revendique haut et fort ce soutien de Fortuna à son égard. À Vorenus qui lui crie « Bonne chance ! », alors qu'il est parti ${ }^{26}$ en toute simplicité exécuter Cicéron selon les ordres d’Octave, il lui répond «C'est mon autre nom ! ».

Lors du combat où Pullo est condamné à mort (1.11), Vorenus vient à son secours, comme dans le texte de César, et c'est par cette scène que se renoue leur amitié brisée, grâce à l'appel au lien indéfectible de la XIII légion (Fig. 34).

${ }^{26}$ Il part accomplir sa mission tranquillement et tout guilleret, au beau milieu d'un pique-nique, proposé à femmes et enfants " parce qu'il avait un petit travail à faire » dans un coin superbe.. C'est le côté pratique de Pullo! 


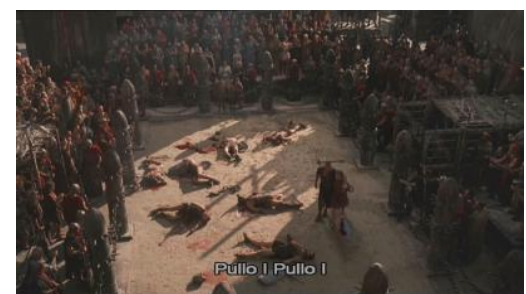

Fig. 34

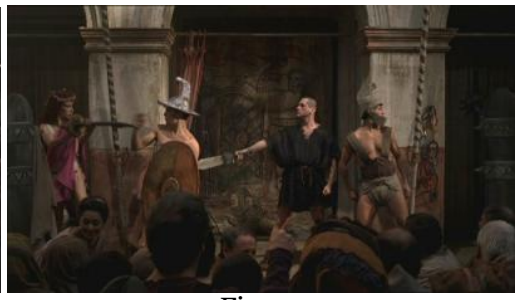

Fig. 35

Bouclant la boucle de la représentation fictionnelle d'une réalité historique, dans le dernier épisode de la première saison, une troupe de comédiens rejoue le combat mythique de Pullo et Vorenus contre le guerrier géant (Fig. 35). Attia et Octavia assistent au spectacle : «Ils sont assez forts, dit Attia avec un air de connaisseuse blasée, mais je les trouve, oui je les trouve beaucoup plus beaux en vrai. » (1.12). Elle commente le rapport entre réel et fictionnel, avec une mise en abyme, au prix d'une étrangeté puisque le réel n'est que le point de vue d'Attia (certes personnage réel mais revu et corrigé pour les besoins de la fiction) sur un épisode fictionnel concernant des personnages réels mais également revus et corrigés pour la fiction.

Il y a donc un jeu sur le média même qui a été choisi, puisque les deux femmes devant le spectacle sont assimilables au téléspectateur regardant la série. Comme Ray Stevenson et Kevin McKidd, Pullo et Vorenus semblent des supers stars, des people à la mode dans tout Rome. Ils sont devenus des héros célèbres, avec des portraits partout, parce qu'ils incarnent " la fraternité, la rédemption ". Pullo, après son combat dans l'arène, quitte l'hôpital, sans même être remis de ses graves blessures, pour ne pas «tout rater », c'est-à-dire pour pouvoir profiter de cette gloire inespérée ${ }^{27}$. Il dit à Vorenus : «On est célèbres, pas vrai ? Des héros ! Des chansons, des spectacles de mime, des dessins sur les murs, hein ? ». Pour Vorenus, ce ne sont que des bêtises, auxquelles il n'accorde aucune importance (il faut dire que César lui offre d'entrer au Sénat, pour récupérer le prestige dont il est désormais auréolé et pour lui servir de garde rapprochée). Pullo a une autre analyse de la situation: quelle opportunité que cette popularité pour séduire les femmes ! Mais il déchante vite : les dessins que l'on fait de lui ne doivent pas trop ressembler à la réalité puisque personne ne le reconnaît spontanément dans la rue et qu'il est obligé, un peu vexé, d'interpeler une jeune fille pour lui demander : « Devine qui je suis ! (1.12).

Comme pour le commentaire d'Attia sur le combat factice, la discussion de Cicéron et Pullo, venu le tuer, nous entraîne dans une discussion uchronique, étrange et vertigineuse, sur le réel et le fictionnel, car c'est Cicéron qui s'exclame « Le célèbre Pullo ! » et qui constate qu'en le

${ }^{27} \mathrm{Il}$ avait déjà connu une première heure de gloire en 1.2 lorsque, toujours prompt à la bagarre, il avait sauvé le premier Marc-Antoine de l'agression des Pompéiens devant le Sénat. 
tuant, Pullo entrera dans l'Histoire, ce qui vexe un peu Pullo qui analysait l'affaire autrement : selon lui, c'est parce que c'était le grand Pullo qui tuait Cicéron que Cicéron entrerait dans l'Histoire ! (2.6)

Les caractères de Pullo et Vorenus sont en contradiction avec le chef qu'ils soutiennent : Pullo ressemble à Marc-Antoine mais c'est un proche d'Octave, et Vorenus ressemble à Octave et soutient Marc-Antoine. Dans la première saison, Vorenus partage les choix du dictateur sur le terrain militaire, mais il est plus réservé sur les idées de l'homme politique et reconnaît la grandeur et la légitimité de Pompée; c'est pourquoi il l'épargne lorsqu'il le reconnaît, lors de sa fuite ${ }^{28}$. Vorenus, désormais aux côtés de César, ne le quitte pas et semble comme son fils, ce que constate avec un peu d'amertume et de jalousie Brutus dans le dernier épisode. Vorenus est même soupçonné un temps par les proches de César d'avoir fait partie du complot, puisqu'il a disparu juste avant l'attaque contre le dictateur: mais la vérité sera dévoilée et le Tu quoque mi fili ne s'appliquera pas à Vorenus, qui n'a pas trahi César volontairement (1.12).

\section{De l'épisode historique à l'exemplum mythique : Pullo et Vorenus dans la Guerre des Gaules de César}

Voici le texte du chapitre XLIV du cinquième livre des Commentaires sur la guerre des Gaules, où Jules César raconte les exploits de deux de ses soldats. Il y est fait allusion à leur valeur guerrière (uirtus), aux ennemis qu'ils affrontent (hostes) et à la rivalité qu'ils éprouvent d'abord l'un envers l'autre (inimicitia ${ }^{29}$ ) :

Il y avait dans cette légion deux centurions d'une très grande bravoure, qui approchaient des premiers grades, Titus Pullo et Lucius Vorenus. C'était entre eux un perpétuel contentieux à qui passerait avant l'autre, et chaque année la question de l'avancement les mettait en violent conflit. Ainsi Pullo, au moment où l'on se battait avec le plus d'acharnement au pied des remparts, s'écria-t-il : « Pourquoi hésites-tu, Vorenus ? Quel grade attendstu en récompense de ta bravoure ? C'est ce jour qui décidera du contentieux entre nous. " Après avoir prononcé ces mots, il s'avance hors des remparts et, là où les ennemis gaulois lui semblent les plus nombreux, il fonce.

\footnotetext{
${ }^{28}$ Choix qui s'avèrera malheureux car au lieu d'être jugé dignement comme tout citoyen romain s'il avait été fait prisonnier par des partisans de César, il sera exécuté par un traitre à la solde des Égyptiens, décapité à peine arrivé sur le sol.

${ }^{29}$ Le mot inimicus employé pour Pullo et Vorenus en fin de texte indique que ce sont des ennemis sur un plan privé, à la différence du mot hostis, utilisé à plusieurs reprises dans le texte, le plus souvent pour désigner globalement les assaillants gaulois du point de vue romain et une fois pour qualifier Pullo, du point de vue gaulois. César ne raconte pas si cette rivalité s'est ensuite muée en amitié.
} 


\begin{abstract}
Vorenus ne reste pas davantage retranché, mais, redoutant le jugement de tout le monde, il s'élance à sa suite. Dès qu'il ne reste que peu de distance entre eux, Pullo jette son javelot sur les ennemis et en atteint un, qui s'était détaché de la troupe pour courir en avant ; transpercé, mourant, les autres le couvrent de leurs boucliers, cependant que, tous à la fois, ils lancent leurs traits contre leur ennemi romain et l'empêchent d'avancer. Le bouclier de Pullo est transpercé et le javelot se plante dans le baudrier : du coup, le fourreau est dévié, sa main droite est ralentie, tandis qu'il cherche à sortir son épée ; et c'est alors qu'il est dans cette fâcheuse position que les ennemis l'enveloppent. Son rival Vorenus accourt et lui vient en aide, puisqu'il est dans l'embarras. Aussitôt, toute la troupe se tourne vers lui et se détourne de Pullo : ils pensent que le javelot l'a tué. Vorenus, avec son épée, lutte corps à corps, en tue un, chasse un peu vers l'avant les autres ; mais, emporté par son ardeur, il se précipite vers un lieu en contre-bas et s'effondre. C'est à son tour d'être enveloppé, mais Pullo lui porte secours, et ils rentrent tous deux au camp, sains et saufs, ayant tué un bon nombre d'hommes et s'étant couverts de gloire. Durant cette lutte et ce combat, la Fortune agit de telle sorte envers l'un et l'autre que chacun aida et sauva son rival, et qu'il fut impossible de décider auquel des deux plutôt qu'à l'autre il semblait qu’il fallait accorder le prix de la bravoure ${ }^{30}$.
\end{abstract}

Nous avons ici une aristeia, du mot grec qui signifie «l'excellence », c'est-à-dire de hauts faits militaires, comme dans l'Iliade d'Homère. Cette anecdote historique est analysable comme un exemplum, racontant de manière allégorique - et pour servir à l'édification - la uirtus du soldat romain, son courage, sa valeur guerrière, son héroïsme, sa bravoure, son sentiment patriotique. La présence de deux soldats pourvus de ces mêmes qualités sert à généraliser l'éloge à tout soldat romain héroïque, sans que cela fasse de lui un héros individualisable, tel Achille ou Hector. C'est un récit rapide, dynamique, avec une succession de verbes d'action, dont certains au présent de narration, permettant de plonger davantage le lecteur dans la bataille. Est-ce une anecdote militaire ou un conte initiatique? Les premiers mots latins sont Erant in ea legione fortissimi uiri centuriones... ( « Il y avait dans cette légion deux centurions d'une très grande bravoure... ») : ils n'ont qu'une attache lâche avec le texte précédent par le biais de l'anaphorique $e a$. L'agencement de cette séquence narrative se fait donc par enchâssement ; mais, linguistiquement et narrativement, rien n'empêcherait la séquence d'apparaitre ailleurs. Ce récit d'un acte de bravoure durant un siège n'est pas circonstancié et aurait pu être « cousu » ailleurs car, même s'ils ont sans doute vraiment existé, rien ne les relie précisément dans le texte à la légion du légat Quintus Cicéron, frère de l'orateur Marcus Tullius. Par sa forme même, proche du «Il était une fois ", et son caractère quasiment autonome, ce texte fonde une autre temporalité chez César.

${ }^{30}$ Jules César, Guerre des Gaules, t. II, Livres V-VIII, éd. L.-A. Constans, rev. et corr. A. Balland, coll. « CUF », Paris, Les Belles Lettres, 2000. Traduction personnelle du passage. 
Pourquoi insérer ce récit? Dans le livre V, César utilise l'épisode précédent du drame de la mort du légat Titurius Sabinus et l'anecdote Pullo-Vorenus pour accroître une matière qui n'était pas assez riche en 54 a.C. ${ }^{11}$ et créer une diversion dans l'esprit du lecteur en provoquant une disjonction des faits, au beau milieu du récit du siège qui tourne au désavantage de Quintus Cicéron. En effet, de retour de Bretagne, les troupes prennent leurs quartiers d'hiver dans diverses cités de la Gaule ; le manque de provisions conduit César à prendre une décision lourde de conséquence : répartir ses légions dans plus de cités que d'habitude. Ils sont en Gaule belge (donc bien loin d'Alésia) ${ }^{32}$. Des Gaulois, de la tribu des Éburons, sous le commandement de leur chef Ambiorix, massacrent les cohortes romaines commandées par les légats Q. Titurius Sabinus et L. Aurunculeius Cotta. Porté par sa victoire, Ambiorix arrive à convaincre une autre tribu, les Atuatuques, de se soulever puis les Nerviens se joignent à eux pour attaquer le camp de Quintus Cicéron ; les Nerviens tentent une négociation avec le légat, qui refuse: le siège du camp commence. Les Romains reprennent un peu le contrôle en faisant pleuvoir des pierres sur leurs assaillants et en mettant le feu à l'une des tours mobiles. C'est alors qu'est introduite la courte digression sur Pullo et Vorenus. César reprend ensuite son récit et évoque les difficultés croissantes que Cicéron et ses troupes ont pour soutenir le siège. Après bien des tentatives infructueuses, un message de détresse parvient enfin à César, qui vient secourir Cicéron. Il n'était donc pas présent lors de l'acte de bravoure de ces deux soldats ; le récit qu'il en fait est donc vraisemblablement écrit en fonction de ce qui lui a été raconté par le légat Cicéron, qui a fait l'éloge de ses hommes.

César a inséré cet épisode dans un but précis, asseoir son prestige de vainqueur de la guerre des Gaules pour mieux marquer des points face à Pompée, au début de la guerre civile. Il ne faut donc pas chercher dans son récit la réalité historique: cet exemplum est déjà une réécriture de l'Histoire à des fins politiques et stratégiques. On peut alors se demander ce qui, dans la conception historique des Romains, différente de la nôtre, autorise un tel récit, et ce qui, dans la structure narrative du texte, permet l'édification.

Pourquoi la vision de l'Histoire de César laisse-t-elle en effet un droit à la fiction historique ? Il faut préciser, tout d'abord, que nous avons, nous modernes, une conception de l'Histoire comme science qui s'éloigne de sa conception souvent très romanesque chez les historiens de l'Antiquité. Selon Marcus Tullius Cicéron, dans le traité De Oratore, un bon historien est un écrivain qui sait dépasser le simple stade du récit des faits bruts (narratio) et la présentation brute du matériel historique (les

${ }^{31}$ Contrairement à ce qui est indiqué au début de l'épisode 1 , le récit autour de Pullo-Vorenus n'a pas eu lieu durant la bataille finale d'Alésia en 52 a.C. mais durant le siège du camp de Quintus Cicéron en 54 a.C. Pour des raisons d'efficacité scénaristique, le récit qui a eu lieu durant une obscure escarmouche a été rattaché à une période plus illustre.

${ }^{32}$ Les Belges sont de redoutables combattants, comme nous l'apprend le début de La Guerre des Gaules (I, i, 3), qui fournit l'argument à Astérix chez les Belges. 
fundamenta qu'une documentation a permis d'établir), pour arriver à créer un ouvrage travaillé (opus ornatum), résultant de l'exaedificatio ou de l'exornatio, c'est-à-dire de cette capacité à construire, à transformer, à embellir le fond en lui donnant une forme : le bon historien ne doit pas être simplement narrator rerum mais exornator rerum ${ }^{33}$. Pour l'orateur, le premier à avoir écrit des théories sur le genre historique, l'Histoire n'est qu'une branche de la rhétorique : elle a donc affaire au vraisemblable, non au vrai. D'où l'éloge que Cicéron fait des Commentaires de César, dont la qualité littéraire lui semble indéniable :

Ses Commentaires, dis-je, sont tout à fait excellents ! Ils sont nus, vont droit au fait, ont une grâce sans aucun apprêt oratoire, comme un corps dépouillé de son vêtement. En voulant fournir des matériaux aux historiens futurs, il a peut-être fait plaisir à des lourdauds, qui seront tentés de friser tout cela au petit fer. Mais aux gens sensés, il a ôté l'envie d'écrire ; car, dans l'histoire, la brièveté élégante et lumineuse est ce qu'il y a de plus agréable 34 .

Parmi les trois principales formes d'écriture historique en prose 35 , César choisit donc les commentarii mais il met en forme ses notes et s'arrange pour ne pas trop faire ressortir ses incertitudes et ses défaites (voire pour les dissimuler, sauf si elles servent un plan plus vaste), tout cela sans mentir : présenter les faits à son avantage, c'est l'art du bon rhéteur. Il donne donc une dimension littéraire à cette prise de notes, surtout dans certains développements qu'il soigne tout particulièrement (épisodes, discours et tableaux), en procédant à un embellissement rhétorique et à l'amplification. Pour ce faire, il compile des documents issus de plusieurs sources: ses propres rapports au Sénat après chaque campagne et sa correspondance officielle et privée, qui repose sur ses souvenirs personnels, éventuellement soutenus par des notes ${ }^{36}$, ainsi que les rapports que ses lieutenants lui ont transmis et qui ne sont pas rendus publics avant la diffusion qu'en fait César dans son œuvre, compléments indispensables chaque fois qu'il n'a pas assisté personnellement aux faits. Il rassemble ensuite les dossiers, les remanie, s'occupe des ligatures, des coupes, des

\footnotetext{
33 Alain Malissard, "L'histoire : écriture ou vérité ? À propos de Pline (Ep., 6, 16 et 20) et de Tacite (Ann., 14, 3-9). ", Mélanges Pierre Lévêque, 5. Anthropologie et société, éd. M.M. Mactoux et E. Geny, Paris, Les Belles Lettres, 1990 [p. 227-243].

34 Marcus Tullius Cicéron, Brutus, t. 7, éd. et trad. J. Martha, Paris, Les Belles Lettres, 19603, lxxv, § 262.

35 Commentarii : notes prises au jour le jour sur un événement que l'on vit ou auquel on participe. C'est un « carnet de notes », un aide-mémoire, qui n'a normalement aucune ambition littéraire. Annales libri : issus de la rédaction officielle des actes des pontifes, ces livres relatent des événements année après année (que ce passé soit très lointain, la fondation de Rome par Tite-Live, ou proche, la fin de la vie de Néron par Tacite). Ils suivent le cadre annuel des magistratures, des prodiges, etc. Historiae, héritières des Enquêtes du Grec Hérodote: événements toujours proches de l'époque de l'auteur, parfois même contemporains. Le cadre chronologique est moins strict: il s'agit d'un travail de synthèse sur une période précise, un événement donné (la conjuration de Catilina par Salluste). C'est le genre le plus élaboré des trois. ${ }^{36}$ Ces deux premiers éléments constituent la « charpente », le canevas des Commentaires.
} 
additions, des précisions, des introductions et des conclusions. Bref, il donne chair au squelette et les retravaille jusqu'à obtenir l'opus ornatum qui provoque l'émerveillement de Cicéron. Bien sûr, cela ne va pas sans provoquer quelques distorsions de la réalité objective. On parle désormais, à la suite des travaux de Michel Rambaud, de la " déformation historique " de César, ou, pour nuancer, de sa tendance à " l'escamotage historique 37 ", plutôt que de celui de la synthèse historique au sens moderne. La pseudoobjectivité permet à César, dans le récit d'une bataille dont certains détails sont tus (comme la localisation exacte, par exemple, d'Alésia ou de Gergovie) de mettre en place des sites et des batailles «standard » qui servent surtout à mettre en avant la stratégie du général. Il est parfois difficile, à la première lecture d'un épisode, de savoir si César raconte une défaite ou une victoire car les revers sont gommés et les victoires amplifiées. L'emploi du discours indirect, qui présente l'apparence de l'objectivité, recèle en fait une subjectivité accrue car tout est écrit selon le point de vue de César. Les études autour de César ont connu une réorientation importante avec le dévoilement de cette technique littéraire par Michel Rambaud, dont l'analyse est partagée, dans la fiction, par le chef des Gaulois Abraracourcix dans Astérix chez les Belges ${ }^{38}$, dans la polémique qui l'oppose au chef belge :

\section{GUEUSELAMBIX. JULES CÉSAR NE DIT JAMAIS DE CARABISTOUILLES ! NOUS SOMMES LES PLUS BRAVES!

ABRARACOURCIX. JULES CÉSAR EST LE PLUS GRAND CARABISTOUILLEUR DU MONDE, ET LES PLUS BRAVES C'EST NOUS !}

Et si l'on regarde de près l'épisode du siège d'Atuatuca dans son ensemble, dans lequel s'insère la digression sur Pullo et Vorenus, on note qu'il a tous les ingrédients d'un bon western américain, mêlant suspense et happy end, selon Paul Marius Martin :

rien n’y manque, ni le chef épuisé mais responsable, ni les défenseurs obstinés, ni la surenchère héroïque, ni la barbarie des 'sauvages', ni l'arrivée tant attendue des 'tuniques bleues' 39.

Car, effectivement, César se doit d'arriver toujours à temps, comme la cavalerie ! Et il se dégage de ces chapitres une morale, notamment dans le récit de la mort des légats Sabinus, Cotta et dans celle du porte-étendard :

\footnotetext{
${ }^{37}$ Michel Rambaud, La déformation historique chez César, coll. « Collection d'études anciennes, Paris, Les Belles Lettres, 1966², p. 111. Voir aussi René Martin et Jacques Gaillard, Les Genres littéraires à Rome, Paris, Nathan, Scodel, 1990, p. 116-119.

${ }^{38}$ Albert Uderzo et René Goscinny, Astérix chez les Belges, Paris, Dargaud, 1979, p. 22. Nous respectons le lettrage en majuscules, qui indique que les personnages, en colère, crient. 39 Paul Marius Martin, La Guerre des Gaules, La Guerre civile. César, coll. "Les textes fondateurs ", Paris, Ellipses-Marketing, 2000, p 48.
} 
Il y a un palmarès du courage mais aussi une leçon sur l'art d'accepter les risques, de choisir entre deux chances. Dans cette perspective, l'épisode de Pullo et de Vorénus, sans importance pour le déroulement de l'action principale, prend valeur d'apologue. César veut montrer non seulement qu'il $\mathrm{y}$ a des braves dans son armée mais qu'à son jugement, conforme à un épicurisme pratique, l'homme brave est celui qui choisit le risque assorti d'éventuels profits : Pullo et Vorenus affrontent la mort, mais en combattant l'ennemi, ce qui est leur devoir militaire, et avec l'espoir, pour chacun, d'être débarrassé de son rival et d'atteindre ainsi les primi ordines. Défendre sa carrière, sa vie ou son honneur en servant l'armée, telle paraît être la règle de cet épicurisme militaire ${ }^{40}$.

En outre, on constate que l'anecdote historique est analysable comme un texte narratif très bien construit, qui repose sur le mythe des jumeaux guerriers et ennemis (Romulus et Remus, Caïn et Abel, Seth et Osiris, etc.), qui se changent ensuite en Castor et Pollux, donnant leur vie pour sauver l'autre chaque fois que nécessaire. En voici le schéma narratif41 :

\begin{tabular}{|c|l|l|}
\hline \multirow{4}{*}{ SITUATION INITIALE } & $\begin{array}{l}\text { présentation des héros (noms et statut dans la légion); } \\
\text { rivalité comme principe moteur. } \\
\text { Les héros apparaissent dans leur « maison » (ici leur } \\
\text { camp), avec une interdiction l'implicite d'en sortir sans } \\
\text { ordre }\end{array}$ \\
\hline $\begin{array}{c}\text { ÉLEMENT } \\
\text { PERTURBATEUR OU } \\
\text { COMPLICATION }\end{array}$ & combat violent près des remparts \\
\hline \multirow{5}{*}{ PERIPETIES } & Déséquilibre & $\begin{array}{l}\text { transgression (aux sens } \\
\text { propre et figuré) de Pullo, } \\
\text { qui, de sa propre } \\
\text { initiative, sort du rang } \\
\text { pour franchir le rempart } \\
\text { et qui se jette seul dans la } \\
\text { mêlée des ennemis }\end{array}$ \\
\cline { 2 - 3 } & Action réparatrice & $\begin{array}{l}\text { Vorenus se précipite } \\
\text { aussi }\end{array}$ \\
\cline { 2 - 3 } & Déséquilibre & $\begin{array}{l}\text { réussite puis échec } \\
\text { Pullo en danger }\end{array}$ \\
\cline { 2 - 3 } & Action réparatrice & Vorenus lui vient en aide \\
\cline { 2 - 3 } & Déséquilibre & $\begin{array}{l}\text { réussite puis échec } \rightarrow \\
\text { Vorenus en danger }\end{array}$ \\
\cline { 2 - 3 } & Action réparatrice & Pullo lui vient en aide \\
\hline
\end{tabular}

$4^{40}$ César, Bellum Gallicum. Liber quintus (La Guerre des Gaules, livre V), éd. M. Rambaud, coll. « Erasme », Paris, PUF, 1974, p. 40.

${ }^{41}$ Jean-Michel Adam, Les Textes types et prototypes. Récit, description, argumentation, explication et dialogue, coll. " Fac linguistique », Paris, Nathan Université, $1997^{4}$ (2001).

Vladimir Propp, Morphologie du conte, coll. « Points essais », Paris, Seuil, 1970. 
Tabl. 2

Le schéma narratif met en évidence un récit cyclique, reposant sur une symétrie entre situation initiale et situation finale.

Le tout apparait, pour l'armée de César, comme un récit fondateur, auquel même une intervention surnaturelle ne manque pas. La dernière phrase du texte attribue à la déesse Fortuna le rétablissement du parfait équilibre entre les deux héros. Le récit a donc une fonction évaluative : il rappelle aux lecteurs le point de vue de César sur la Fortuna, résumé plus loin par un simple proverbe (au présent de vérité générale et non avec des passés comme dans le récit de l'aristeia de Pullo et Vorenus) : "Le pouvoir de la Fortune est grand dans tous les domaines, et tout particulièrement dans les événements militaires ${ }^{42} »$. Le processus est différent du point de vue de la polyphonie : la fin de l'épisode de Pullo-Vorenus laisse toujours entendre uniquement la voix de César, alors qu'un proverbe est un fragment polyphonique, qui donne à entendre une voix collective et anonyme. L'adverbe qui ouvre la dernière phrase latine souligne la morale de cette histoire et laisse place au surnaturel avec Fortuna, le sujet du verbe 43 . Plusieurs mots avec des nuances différentes indiquent la binarité dans les deux dernières phrases texte, réunissant nos deux rivaux qui ne forment plus qu'une entité linguistique par le biais des pronoms44. Même leurs actions ne peuvent s'exprimer que par des synonymes qui s'imbriquent entre eux ${ }^{45}$. Paul Marius Martin a étudié l'importance de la Fortune dans le corpus césarien. Sa conclusion est que «l'évocation de la fortuna concerne, dans le Bellum Gallicum, essentiellement la fortuna belli », c'est-à-dire l'incertitude de la fortune à la guerre ; et le terme est employé «soit (pour) des ennemis, soit (pour) des lieutenants de César plus ou moins heureux. Tout se passe comme si eux seuls avaient à subir,

\footnotetext{
${ }^{42}$ César, La Guerre des Gaules, vi, 30, 2 (Multum cum in omnibus rebus, tum in re militari potest Fortuna).

Voir également les derniers mots de Brutus, selon Dion Cassius (L'Histoire romaine, 47, 49): "Vertu, tu n'étais qu'un nom. Je te cultivais comme une réalité et tu étais l'esclave de la Fortune », citées dans le docu-fiction Le Destin de Rome 2 - Rêves d'empire.

43 Le verbe uersauit ("a placé »), dont les deux hommes sont COD, est le fréquentatif de uertere, «tourner »; il signifie donc " tourner et retourner », ce qui convient bien aux vicissitudes de Fortuna, de sa roue et aux péripéties de l'action détaillées plus haut, même lorsque la déesse Fortuna est représentée sur une sphère et non à côté d'une roue.

${ }^{44}$ Citons ambo ( tous les deux à la fois »), utrumque ( l'un et l'autre »), alter alteri («l'un des deux à l'autre des deux ») et uter utri (« lequel des deux auquel des deux »).

45 « Ils se secoururent et se sauvèrent mutuellement la vie » (auxilio salutique esset).
} 
pour le meilleur ou pour le pire, la fortuna belli ${ }^{46}$ ». César, lui, sait se concilier les faveurs de Fortuna, dans La Guerre des Gaules, ou reconnaître, dans Rome, ceux qu'elle favorise. Cette présence de Fortuna dans le texte a été respectée dans la série puisque la déesse protège Vorenus et Pullo, comme César l'avait fait remarquer à Antoine, et que c'était même la roue de la Fortune qui ouvrait le générique.

Pourquoi les scénaristes de la série Rome ont-ils choisi ce texte comme point de départ pour leur série ? Parce qu'il est bien écrit, ciselé par César sur le plan narratif et stylistique, qu'il se détache de l'ouvrage comme un morceau de choix, qu'il fait appel à l'imaginaire épique; mais aussi parce qu'il n'est qu'un fragment de vie de personnages peu ordinaires mais qui sont en marge de l'Histoire, et qui permettent donc l'invention ; parce que ces guerres de César en Gaule sont un écho à la guerre de Bush en Irak.

Mais les scénaristes avaient-ils le droit de détourner le texte historique de César? Nous répondrons doublement par l'affirmative, d'abord parce que Rome n'est pas un documentaire historique, c'est une fiction, et ensuite parce que cette fiction repose sur un texte qui est luimême une reconstruction littéraire d'une réalité qui nous échappe en partie, comme nous avons essayé de le montrer dans le point précédent de notre réflexion. Dans l'épisode écrit par César, c'est le mythe du superhéros qui fonctionne à plein; dans Rome, il est déconstruit, pour s'intéresser davantage à Clark Kent qu'à Superman. Car la série commence avec la fin de la guerre, la fin de leur rôle bien défini de soldats et la difficulté de revenir dans la vie de tous les jours et de retrouver une place dans leur famille et dans la société.

${ }^{46}$ Paul Marius Martin, "Pourquoi écrire la Guerre civile quand on est César ? ", Cahiers des anciennes, xlvi, 2009, § 36-37, mis en 